\title{
Amniotic Fluid Lecithin to Sphingomyelin Ratio of 3.5 and Fetal Pulmonary Maturity
}

\author{
E. B. OLSON, JR., ${ }^{(33)}$ S. N. GRAVEN, AND R. D. ZACHMAN
}

Department of Pediatrics, University of Wisconsin Medical School, Madison, Wisconsin, USA

\section{Extract}

The clinical outcome of 118 pregnancies assessed by amniotic fluid lecithin to sphingomyelin concentration $(\mathrm{L} / \mathrm{S})$ ratio within 2 days of delivery is presented. It has been observed that the critical L/S ratio for pulmonary function is 3.5 in that no infant born with an $\mathrm{L} / \mathrm{S}$ ratio greater than 3.5 has had respiratory distress syndrome (RDS). Below an $\mathrm{L} / \mathrm{S}$ ratio of 3.5 , the risk and severity of RDS is inversely related to the $\mathrm{L} / \mathrm{S}$ ratio.

An $L / S$ ratio $<3.5$ has the same prognostic value in infants born between 30 and 34 weeks gestation as it has in infants born between 35 and 38 weeks gestation. Under these conditions the relation between $\mathrm{L} / \mathrm{S}$ ratio and $\mathrm{RDS}$ is independent of gestational age.

\section{Speculation}

It has not been demonstrated directly to what extent the developing fetal lungs actually contribute to the amniotic fluid phospholipids. We feel that the amniotic fluid L/S ratio indeed serves as an index of pulmonary maturation, but that there are significant misconceptions about the term "L/S ratio" which have contributed to errors in performing and interpreting this test.

After the initial suggestion by Graven (15) that amniotic fluid phospholipid concentrations showed a correlation with RDS, over 80 studies have appeared in the literature dealing with the analysis of amniotic fluid phospholipids as an indicator of fetal lung maturation. The most influential report appeared in 1971 by Gluck et al. (12) in which amniotic fluid lecithin concentration was correlated with increasing gestational age.

These authors proposed that: (1) both sphingomyelin and lecithin in amniotic fluid originate in the fetal lungs; (2) the rise in lecithin concentration coincides with the appearance of a competent pulmonary surfactant system; (3) to avoid any variations in the communication between the fetal lungs and the amniotic fluid, the ratio of lecithin to sphingomyelin concentration should be used as an index of pulmonary maturation. Later, in two widely circulated communications, Gluck and Kulovich presented a table which outlined predicted fetal outcome related to amniotic fluid lecithin to sphingomyelin $(\mathrm{L} / \mathrm{S})$ ratios. This table was the first proposal that an $\mathrm{L} / \mathrm{S}$ ratio greater than 2.0 indicated fetal pulmonary maturation $(13,14)$.

Since its introduction, the $\mathrm{L} / \mathrm{S}$ ratio has been the major method used to predict fetal lung maturity. Experiences using this test have not been all good as reflected by controversy in the scientific literature $(1,3-5,10)$. Some of this disparity has been attributed to differences in methodology (7). It is our feeling that interpretation of the value of the $\mathrm{L} / \mathrm{S}$ ratio as a determinant of lung maturity requires a careful understanding of methods among investigators, and that the critical L/S ratio based on the absolute amounts of the two phospholipids is 3.5 (not 2.0) (20). The purpose of this paper is to report our experiences using the ratio of the concentration of lecithin to the concentration of sphingomyelin to assess fetal lung competency.

\section{MATERIALS AND METHODS}

The techniques involved in analyzing amniotic fluids are lipid extraction followed by thin layer chromatography and quantitation. In our experience some aspects deserve special care.

\section{PREPARATION OF AMNIOTIC FLUID}

Centrifugation of amniotic fluid to be analyzed for pulmonary phospholipids should be a carefully evaluated procedure. Lung washes often contain lamellar structures (at least partially composed of phospholipids of the pulmonary surfactant system). These lamellar structures may sediment when exposed to high gravity. On the other hand, it is well known that lecithin is a ubiquitous component of all membranes; therefore, any cellular contamination represents a source of nonpulmonary surfactant lecithin and should be removed before analyzing for phospholipids of pulmonary origin. In our experience the majority of amniotic fluids do not contain excessive cellular contamination. We simply allow these fluids to settle for a few minutes and remove a supernatant aliquot for extraction. Occasionally an amniotic fluid is contaminated with vernix or blood cells (27); in these cases the amniotic fluid is centrifuged at $500 \times g$ for $2 \mathrm{~min}$ at $0-4^{\circ}$, and the clear supernatant is sampled. Subjection of amniotic fluid to higher gravities for longer periods of time results in a decrease in the amount of phospholipids which can be extracted from the supernatant $(18,23)$. The presence of meconium-stained fluid was occasionally noted. The results of the lipid extractions from these fluids were not included in this evaluation.

\section{LIPID EXTRACTION}

The extraction was done in Pyrex centrifuge tubes (104 by $17.5 \mathrm{~mm}$ ) which fit the SS-34 head of a RC2-B Sorvall centrifuge using no. 328 rubber adaptors. Usually $2.0 \mathrm{ml}$ amniotic fluid were extracted; however, smaller volumes could easily be analyzed simply by scaling down the extraction procedure. One part of methanol was mixed with one part amniotic fluid. Two parts of chloroform were added, a clean 
silicone rubber stopper was inserted and the mixture is vortexed for $1 \mathrm{~min}$. The phases were split by centrifuging at approximately $12,000 \times g$ for $5 \mathrm{~min}$ and the lower chloroform phase was removed. Two more parts of chloroform were vortexed with the remaining upper phase for $1 \mathrm{~min}$, the centrifugation was repeated, and the two chloroform phases were combined in a $100-\mathrm{ml}$ round-bottomed flask. (Two chloroform extractions were performed since it was shown that they were necessary for removing all the extractable phospholipids.) The chloroform was rapidly removed under reduced pressure in a rotary evaporator. Exactly $1.0 \mathrm{ml}$ cold chloroform was added and swirled to wash the extract from the walls of the flask. A $0.9-\mathrm{ml}$ aliquot of this $1.0 \mathrm{ml}$ was then withdrawn and evaporated to dryness under nitrogen. The dried lipid extract was dissolved in $50 \mu \mathrm{l}$ chloroform and from $1-5 \mu \mathrm{l}$ of this were spotted on silica gel-coated glass plates for thin layer chromatography.

\section{CHROMATOGRAPHY, QUANTITATION AND} QUALITY CONTROL

From 2-10 percent of the final lipid extract were spotted in $1-5 \mu \mathrm{l}$ on an approximately $250-\mu \mathrm{m}$ thick layer of silica gel $\mathrm{H}$-coated on to thin glass plates $(8$ by $10 \mathrm{~cm})$ which had been activated in a $110^{\circ}$ oven for at least $30 \mathrm{~min}$ and stored in a dessicator. The plates were developed in $65 / 25 / 4(\mathrm{v} / \mathrm{v} / \mathrm{v})$ chloroform-methanol-water. After allowing the chromatograph solvent to evaporate, the phospholipid spots were developed by charring after spraying the plate with either $50 \%(\mathrm{v} / \mathrm{v})$ sulfuric acid or (more recently) with $1 \%$ potassium dichromate in $70 \%(\mathrm{v} / \mathrm{v})$ sulfuric acid. A more complete description of these charring methods along with the standard curves of lecithin and sphingomyelin visualized under these conditions has been reported (20). Every plate also contained two or three spots with a standard amount of lecithin and sphingomyelin. Specifically, $2.70 \mathrm{nmol}$ lecithin (DL- $\alpha$-lecithin, Schwarz-Mann no. 1230) and $2.76 \mathrm{nmol}$ sphingomyelin (Sigma no. S-3251) on the earlier plates charred with $50 \%$ sulfuric acid, and $2.511 \mathrm{nmol}$ each lecithin (DL- $\alpha$-lecithin, Sigma no. L-8878) and sphingomyelin (Sigma no. S-3251) on more recent plates charred with potassium dichromate in sulfuric acid. These standard solutions were prepared by diluting a known weight of each chromatographically pure phospholipid with chloroform. The concentrations were then confirmed by phosphate analysis (25).

After the phospholipids were visualized and identified, the optical densities of the lecithin and sphingomyelin from each spot were measured using a rapid scanning, integrating, recording densitometer (28). The sensitivity settings of the recorder were changed with each plate in order to maximize the peak obtained from the darkest phospholipid spot on the plate. Therefore, the absolute integrated areas obtained from the same amount of phospholipid varied from plate to plate. This variation was overcome by expressing the integrated area of each optical density peak on an individual plate relative to the average area of the standard sphingomyelin spots on the same plate.

Unknown amounts of lecithin and sphingomyelin were quantitated using the phospholipid standard curves shown in either Figure 1 (50\% sulfuric acid charring) or Figure 2 (dichromate-sulfuric acid charring). In either case, it has been shown previously that the standard curves of lecithin and sphingomyelin do not differ significantly (20). Therefore, for the purpose of quantitation of unknowns, the data from both lecithin and sphingomyelin standard curves was combined into one phospholipid standard curve. As an additional control, a 2.0-ml aliquot from a large pooled amniotic fluid sample was extracted, chromatographed, and quantitated in parallel with each unknown amniotic fluid. If this control sample was not within $10 \%$ of its known mean $\mathrm{L} / \mathrm{S}$ ratio value, the entire assay was repeated.
CLINICAL DATA AND ASSESSMENT OF RDS

During the period from August 25, 1971 to October 22, 1973 , a total of 471 clinically useful amniotic fluid L/S ratios were performed on samples from 386 pregnancies. In 267 cases, follow-up information including the hospital and date of delivery, gestational age assessment (29), and an evaluation of symptoms of respiratory distress was obtained. Of these, nine pregnancies were excluded from the follow-up evaluation. Six were excluded because of fetal death before delivery; the other three are part of an ongoing study involving the use of corticosteroid treatment which may rapidly alter pulmonary maturation. Two of the remaining 258 pregnancies resulted in twins. The fetal outcome in both of these cases was identical and it was decided to regard each of these two cases as a single pregnancy since it is not certain which of the twin's amniotic fluid had been sampled.

Forty-eight of the outcomes had at least minimal indications of respiratory distress. In these instances the mother's and infant's hospital records were obtained and carefully analyzed. The diagnosis and assessment of severity of RDS was made objectively using the criteria outlined by Hobel et al. (17). The cases were divided into three categories based on the pulmonary status outline of Table 1 .

\section{RESULTS}

The pulmonary status categorization of the 258 infants evaluated is presented in Table 2. Notice that seven of the follow-up assessments have some uncertainty associated with their diagnosis. We feel confident that these cases have been correctly categorized; however, even if these seven cases are omitted, the conclusions based on this data would be unaffected.

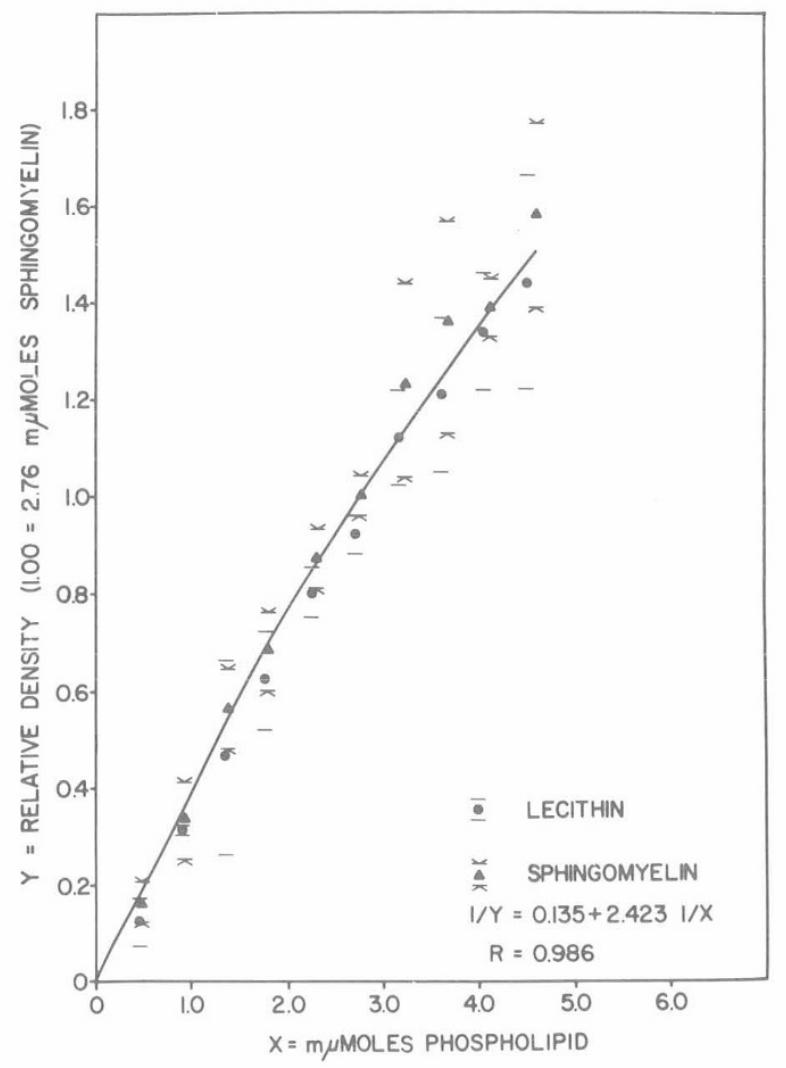

Fig. 1. Relative densities of known amounts of sphingomyelin and dipalmityl lecithin chromatographed on silica gel, sprayed with $50 \%$ (V/V) sulfuric acid, and charred. Brackets represent 95\% confidence limits. The phospholipid standard curve is the result of reciprocal linear regression analysis. 


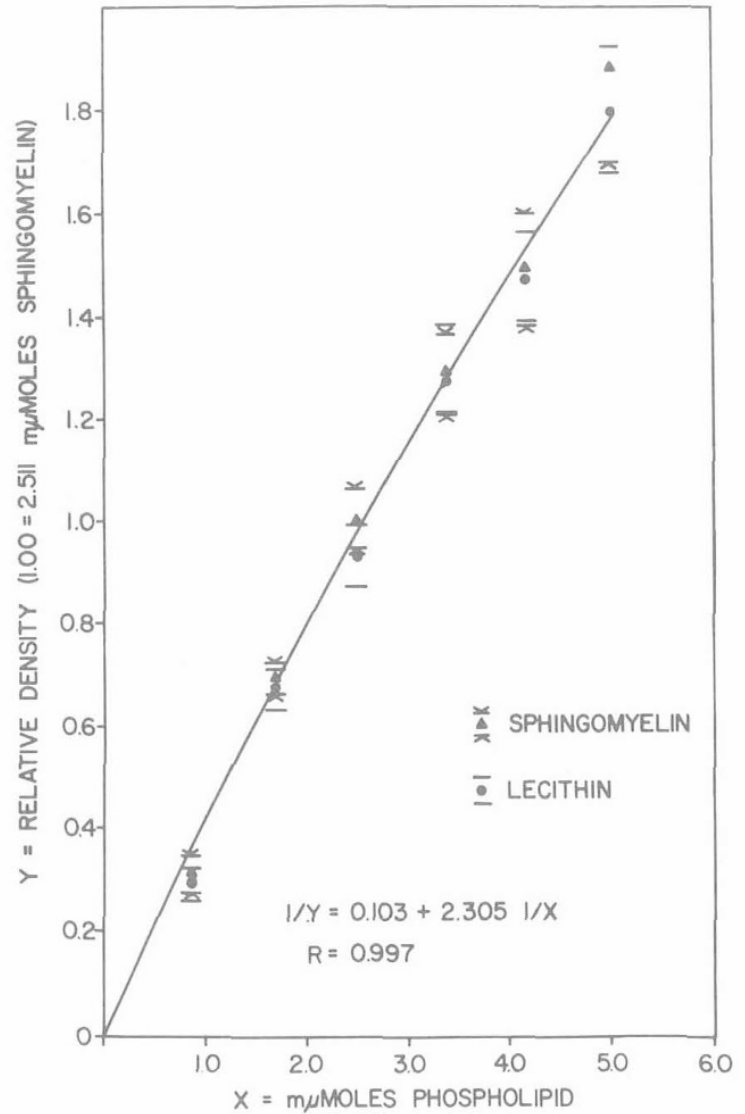

Fig. 2. Phospholipid standard curve calculated by reciprocal linear regression analysis of the relative densities of known amounts of sphingomyelin and dipalmityl lecithin chromatographed on silica gel, and visualized by spraying with $1 \%$ potassium dichromate in $70 \%(\mathrm{~V} / \mathrm{V})$ sulfuric acid and charring. Brackets are $95 \%$ confidence limits.

Table 1. Pulmonary status outline

I. No RDS

A. No RDS

B. ? no RDS; the infant's clinical course manifests some of the criteria of Hobel et al. (17) for RDS. The etiology is clearly not pulmonary surfactant deficiency.

C. Death, no RDS; neonatal death from causes not primarily related to lung function.

II. RDS

A. RDS; clearly due to pulmonary surfactant deficiency.

B. ? RDS; cause of RDS most likely pulmonary surfactant deficiency.

III. RDS $\rightarrow$ death

A. Immature; these cases were assessed at less than 27 weeks gestation (8) and lived only briefly. However, it is assumed that their prematurity included a deficiency of pulmonary surfactant.

B. RDS.

C. ? RDS; there were two cases in this subcategory. In one the hospital record is not complete enough to unambiguously diagnose RDS. In the other, the cause of RDS is most likely pulmonary surfactant deficiency.

Amniotic fluid $\mathrm{L} / \mathrm{S}$ ratios may remain unchanged for several weeks. However, some laboratories, including ours, have observed cases in which amniotic fluid L/S ratios have risen dramatically in a matter of days (3). For optimal prognostic accuracy, the interval between amniocentesis and evaluation of fetal outcome should be as short as possible. Figure 3 shows the intervals between the date of amniocentesis and the date of birth of the 258 babies evaluated. Because of this data, as well as on practical considerations involving the normal time lag between amniocentesis and elective intervention, it was decided to limit all further tabulations to data gathered from the 118 babies born within 2 days of the date of amniotic fluid sampling.

In Table 3 our experience with the amniotic fluid L/S ratio within 2 days of birth and the subsequent pulmonary status of the infant is tabulated. The first column shows the total number of babies in each of seven L/S ratio ranges. Out of this total, the number developing RDS is shown in column 2. The severity of RDS is partially conveyed by the third column

Table 2. Pulmonary status ${ }^{1}$

\begin{tabular}{lc}
\hline \multicolumn{1}{c}{ Category } & No. infants \\
\hline I. No RDS & \\
A. No RDS & 224 \\
B. ? No RDS & 2 \\
C. Death, no RDS & 5 \\
Subtotal & 231 \\
II. RDS & \\
A. RDS & 10 \\
B. ? RDS & 3 \\
Subtotal & 13 \\
III. RDS $\rightarrow$ death & \\
A. Immature $(<27$ wk) & 7 \\
B. RDS & 5 \\
C. ? RDS & 2 \\
Subtotal & 14 \\
Total & 258 \\
\hline
\end{tabular}

${ }^{1}$ RDS: respiratory distress syndrome.

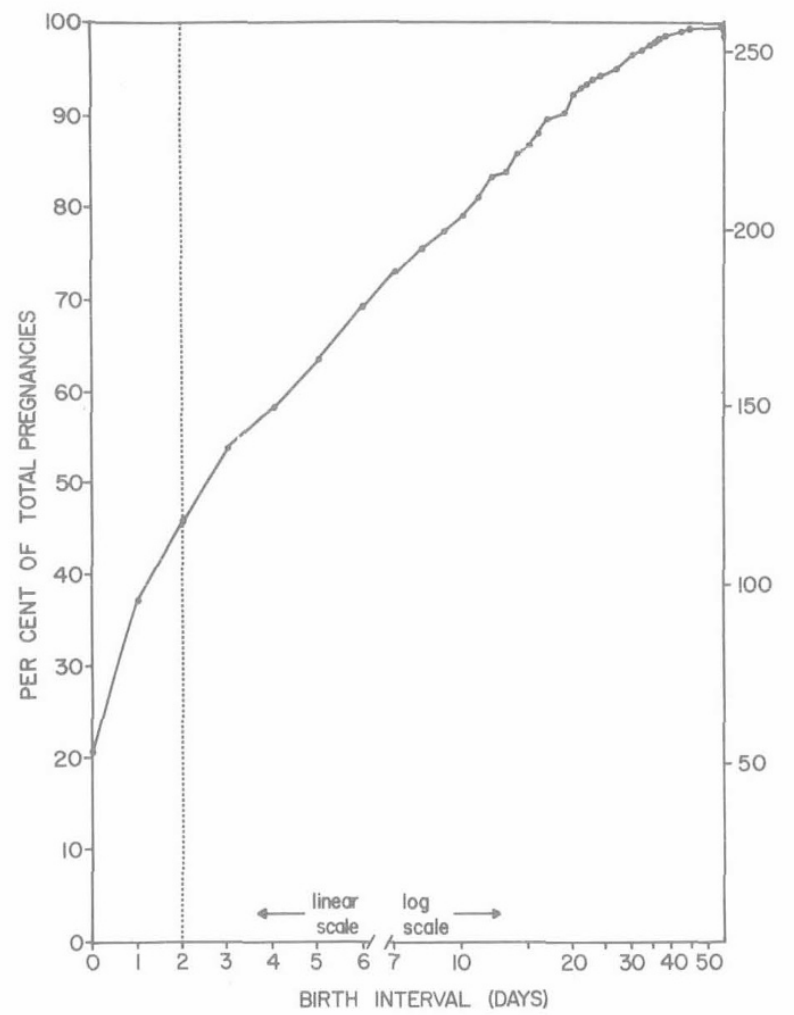

Fig. 3. Interval between amniocentesis and birth date of the 258 infants evaluated. Based on this total experience, the remaining interpretation tables are limited to data from infants born within 2 days of amniocentesis. 
Table 3. $L / S$ ratio range versus pulmonary status: amniocentesis within 2 days of birth ${ }^{1}$

\begin{tabular}{lccc}
\hline L/S ratio & Total & Total with RDS & RDS $\rightarrow$ death \\
\hline$\leqslant 1.0$ & 2 & 2 & 2 \\
$1.1-1.5$ & 3 & 3 & 3 \\
$1.6-2.0$ & 8 & 7 & 4 \\
$2.1-2.5$ & 4 & 2 & 1 \\
$2.6-3.0$ & 11 & 4 & 0 \\
$3.1-3.5$ & 19 & 2 & 0 \\
$>3.5$ & 71 & 0 & 0 \\
\hline
\end{tabular}

${ }^{1}$ RDS: respiratory distress syndrome.

which shows the number of cases in which RDS resulted in neonatal death. In our experience, no infant born with an L/S ratio greater than 3.5 has had RDS and no infant born within 2 days of an $\mathrm{L} / \mathrm{S}$ ratio less than 1.5 has survived. The $\mathrm{L} / \mathrm{S}$ ratio ranges between 1.6 and 3.5 represent a zone where the risk and severity of RDS seems to vary inversely with the L/S ratio. - In Table 4 the pulmonary status of the babies born at a gestational age between 30 and 34 weeks and between 35 and 38 weeks are tabulated so that the clinical outcome relative to the critical L/S ratio of 3.5 can be visualized. This table delineates four populations of infants born with a known L/S ratio: (1) 12 infants between 30 and 34 weeks gestational age with an $\mathrm{L} / \mathrm{S}$ ratio $\leqslant 3.5$; (2) 8 infants between 30 and 34 weeks gestational age with an $\mathrm{L} / \mathrm{S}$ ratio $>3.5$; (3) 22 infants between 35 and 38 weeks gestational age with an L/S ratio $\leqslant 3.5 ;$ (4) 32 infants between 35 and 38 weeks gestation with an $\mathrm{L} / \mathrm{S}$ ratio $>3.5$.

In order to provide a basis for estimating the expected incidence of RDS in the normal population of infants in these two gestational age groups, the pulmonary status of all babies born at this institution during the past 4 years within the 30-34 week and 35-38 week gestational age groups is also included in Table 4. The data from Table 4 is also presented in Table 5 distributed across the range of gestational ages from 30-38 weeks. Using the chi-square probability distribution in Table 4 it is possible to determine whether the incidence of RDS in any of the four populations defined by $\mathrm{L} / \mathrm{S}$ ratio differ significantly from the incidence of RDS in the corresponding control populations.

\section{DISCUSSION}

Our interpretations of amniotic tluid $\mathrm{L} / \mathrm{S}$ ratios are now made on the basis of our experience as summarized in Table 3. However, when we first started to perform L/S ratios we used interpretations published by others $(13,14)$. At that time we assumed that an L/S ratio over 2.0 signified fetal lung maturity and assumed that $\mathrm{L} / \mathrm{S}$ ratio meant the ratio of the concentration of lecithin to the concentration of sphingomyelin in amniotic fluid (12). Our assumptions were incorrect. This was discovered when eight cases, in which babies born after an amniotic fluid L/S ratio greater than 2.0, developed RDS due to pulmonary surfactant deficiency. In six of these cases the $\mathrm{L} / \mathrm{S}$ ratio was also performed using acetone precipitation and the resulting ratios were unchanged within the expected variablity range introduced by this procedure (30). This data was presented to the Society for Pediatric Research and the American Pediatric Society with the recommendation that every laboratory performing this analysis use its own experience with fetal outcomes as its interpretation (26). It was at this time that we learned that L/S ratio is not always the ratio of the concentration of lecithin to the concentration of sphinogomyelin but often the ratio of the density of lecithin visualized to the density of sphingomyelin visualized. Based on our experience (20), we predict that if other laboratories construct standard curves for lecithin and sphingomyelin by whatever visualization method they are using, their $\mathrm{L} / \mathrm{S}$ ratios, based on the absolute amounts or concentrations of these phospholipids, will confirm that an L/S ratio of greater than 3.5 (and not 2.0) corresponds to fetal pulmonary maturation. The standard curves for lecithin and sphingomyelin, using several visualization methods, are available for comparison (20).

A statistical analysis of the data presented in Tables 4 and 5 supports the concept that the $\mathrm{L} / \mathrm{S}$ ratio truly reflects lung maturation irrespective of gestational age. If we direct our attention to Table 4, of the two L/S ratio populations between 30 and 34 weeks gestation, there seems to be a trend toward a lower than "normal" incidence of RDS in the infants with an $\mathrm{L} / \mathrm{S}$ ratio $>3.5(P<0.15)$, even though there is $0 \mathrm{RDS}$ in the $\mathrm{L} / \mathrm{S}$ ratio $>3.5$ group there are not enough infants to provide a significant difference relative to the incidence of RDS in the control group. The results in the $35-38$ weeks age group are statistically stronger. Between these gestational ages the incidence of RDS in the population defined by an L/S ratio $\leqslant 3.5$ is elevated significantly over the "normal" incidence of RDS $(P<0.0001)$. Based on this experience we feel that the amniotic fluid $\mathrm{L} / \mathrm{S}$ ratio corresponds with fetal pulmonary maturation as evidenced by development of respiratory difficulty after birth irrespective of gestational age as originally proposed (12). This finding disagrees with reports of other authors who feel that amniotic fluid L/S ratio correlates with gestational age and not directly with lung maturation $(19,21$, 22).

The distribution of the data presented in Table 4 across the range of gestational ages from $30-38$ weeks is shown in Table 5 . The experience of neonatal outcome versus gestational age, as experienced in this institution over the past 4 years, corresponds very closely with similar data reported previously

Table 4. Pulmonary status relative to gestational age range and $L / S$ ratio of 3.5

\begin{tabular}{lrrrrr}
\hline & \multicolumn{2}{c}{$30-34$ Weeks } & & \multicolumn{2}{c}{$35-38$ Weeks } \\
\cline { 2 - 3 } \cline { 5 - 6 } Population & Total & RDS & & Total & RDS \\
\hline L $/ \mathrm{S} \leqslant 3.5$ & 12 & 4 & & $22^{1}$ & 7 \\
L $/ \mathrm{S}>3.5$ & 8 & 0 & & 32 & 0 \\
Control $^{2}$ & 140 & 39 & & $1,302^{1}$ & 17 \\
\hline
\end{tabular}

${ }^{1}$ Significantly different incidence of RDS (respiratory distress syndrome), $P<0.0001$.

${ }^{2}$ All babies born at SMHMC from July 1, 1968, to June 30, 1972.

Table 5. Pulmonary status relative to gestational age and $L / S$ ratio of 3.5

\begin{tabular}{|c|c|c|c|c|c|c|c|c|c|}
\hline \multirow[b]{2}{*}{ Population } & \multicolumn{9}{|c|}{ Gestational age, wk } \\
\hline & 30 & 30 & 32 & 33 & 34 & 35 & 36 & 37 & 38 \\
\hline \multicolumn{10}{|l|}{$L / S \leqslant 3.5$} \\
\hline Total & 0 & 0 & 3 & 4 & 5 & 10 & 7 & 0 & 5 \\
\hline $\mathrm{RDS}^{1}$ & 0 & 0 & 1 & 2 & 1 & 4 & 2 & 0 & 1 \\
\hline \multicolumn{10}{|l|}{$\mathrm{L} / \mathrm{S}>3.5$} \\
\hline Total & 2 & 1 & 1 & 1 & 3 & 4 & 13 & 8 & 7 \\
\hline RDS & 0 & 0 & 0 & 0 & 0 & 0 & 0 & 0 & 0 \\
\hline \multicolumn{10}{|l|}{ Control $^{2}$} \\
\hline Total & 22 & 19 & 24 & 33 & 42 & 53 & 185 & 337 & 727 \\
\hline RDS & 9 & 8 & 11 & 5 & 6 & 6 & 4 & 1 & 0 \\
\hline
\end{tabular}

${ }^{1}$ Respiratory distress syndrome.

${ }^{2}$ All babies born at SMHMC from July 1, 1968, to June 30, 1972. 
by others $(2,9,24)$. This reinforces the assumption that our control population is valid. Notice that one baby of 38 weeks' gestational age with an $\mathrm{L} / \mathrm{S}$ ratio $\leqslant 3.5$ developed RDS. This case does not appear in our control data since the baby was born outside this hospital; cases of RDS at this gestational age are rare, but do occur $(9,24)$.

In conclusion, we feel that the amniotic fluid $\mathrm{L} / \mathrm{S}$ ratio is a useful method for predicting the development of respiratory difficulty after birth. However, the term "L/S ratio" does not always mean the concentration of lecithin divided by the concentration of sphingomyelin, but may simply mean the spot density of lecithin relative to the spot density of sphingomyelin after thin layer chromatography. It is only through universal adoption of standard, comparable techniques that this test can be used to its full potential. In the interests of unambiguous agreement we strongly recommend that the term L/S ratio be understood to mean the ratio of absolute amount of lecithin to absolute amount of sphingomyelin. The critical L/S ratio based on phospholipid concentration is 3.5. In situations where it is not possible to convert to the measurement of phospholipid concentration, authors should state explicitly what the term "L/S ratio" means in their context.

\section{REFERENCES AND NOTES}

1. Arvidson, G., Erkelund, H., and Astedt, B.: Phospholipid composition of human amniotic fluid during gestation and at term. Acta Obstet. Gynecol. Scand., 51: 71 (1972).

2. Avery, M. E.: Personal communication.

3. Bhagwani, S. G., Fahmy, D., and Turbull, A. C.: Prediction of neonatal respiratory distress by estimation of amniotic-fluid lecithin. Lancet, $i$ : 159 (1972).

4. Biezenski, J. J.: Amniotic fluid phospholipids in early gestation. Obstet. Gynecol., 41: 825 (1973).

5. Boehm, F. H., Srisupundit, S., and Ishii, T.: Lecithin/sphingomyelin ratio and a rapid test for surfactant in amniotic fluid: A comparsion. Obstet. Gynecol., 41: 829 (1973).

6. Bryan, R.: Validity of the lecithin-spingomyelin (L/S) ratio for amniotic fluid containing blood. Clin. Chem., 18: 1551 (1972).

7. Charnock, E. L., and Doershuk, C. F.: Developmental aspects of the human lung. Pediat. Clin. N. Amer., 20: 275 (1973).

8. Dubowitz, L. M. S., Dubowitz, V., and Goldberg, C.: Clinical assessment of gestational age in the newborn infant. J. Pediat. 77: 1 (1970).

9. Fedrick, J., and Butler, N. R.: Hyaline-membrane disease. Lancet, ii: 768 (1972).

10. Freeman, R. K., Donald, I. R., Goblesman, U. E., and Chan, A.: Clinical experience with amniotic fluid lecithin/spingomyelin ratio as a measure of fetal pulmonary maturity (Abstract). Obstet. Gynecol., 39: 630 (1972).

11. Gibbons, J. M., Huntley, T. E., Joachim, E., Ruperto, S., and Corral, A. G.: Amniotic fluid analysis for fetal maturity: effect of maternal blood contamination (Abstract). Obstet. Gynecol. 39: 631 (1972).

12. Gluck, L., Kulovich, M. V., Borer, R. C., Jr., Brenner, P. H., Anderson, G. G., and Spellacy, W. N.: Diagnosis of the Respiratory Distress Syndrome by Amniocentesis. Amer. J. Obstet. Gynecol., 109: 440 (1971).

13. Gluck, L., and Kulovich, M.: Procedure for the Intrauterine Assessment of pulmonary maturity by the phospholipids in amniotic fluid. Personal communication, 1971.
14. Gluck, L., and Kulovich, M.: Procedure for the intrauterine assessment of pulmonary maturity by the phospholipids in amniotic fluid: Revised. Personal communication, 1971.

15. Graven, S. N.: Phospholipids in human and monkey amniotic fluid (Abstract). Pediat. Res., 2: 318 (1968).

16. Harding, P., Possmayer, F., Milne, K., Jaco, N.-T., and Walters, J. H.: Amniotic fluid phospholipids and fetal maturity. Amer. J. Obstet. Gynecol., 115: 298 (1973).

17. Hobel, C. J., Possmayer, F., Milne, K., Jaco, N.-T., and Walters, J. $\mathrm{H}$.: Early versus late treatment of neonatal acidosis in low-birthweight infants: relation to respiratory distress syndrome. J. Pediat., 81: 1178 (1972).

18. Lindbach, T., and Frantz, T.: Personal communication.

19. Nakamura, J., Roux, J. F., Brown, E. G., and Sweet, A. Y.: Total lipids and the lecithin-sphingomyelin ratio of amniotic fluid: An antenatal test of lung immaturity? Amer. J. Obstet. Gynecol., 113: 363 (1972).

20. Olson, E. B., Jr., and Graven, S. N.: Comparison of visualization methods used to measure the lecithin/sphingomyelin ratio in amniotic fluid. Clin. Chem., 20: 1408 (1974).

21. Roux, J. F., Nakamura, J., Brown, E., and Sweet, A. Y.: The lecithin-sphingomyelin ratio of amniotic fluid: An index of fetal lung maturity? Pediatrics, 49: 464 (1972).

22. Roux, J. F., Nakamura, J., and Brown, E. G.: Further observations on the determination of gestational age by amniotic fluid analysis. Amer. J. Obstet. Gynecol., 116: 633 (1973).

23. Shelly, S. A., Takagi, L. R., and Balis, J. V.: Assessment of surfactant activity in amniotic fluid for evaluation of fetal lung maturity. Amer. J. Obstet. Gynecol., 116: 369 (1973).

24. Usher, R. H., Allen, A. C., and McLean, F. H.: Risk of respiratory distress syndrome related to gestational age, route of delivery, and maternal diabetes. Amer. J. Obstet. Gynecol. 111: 825 (1971).

25. Weinhold, P. A., and Villee, C. A.: Phospholipid metabolism in the liver and lung of rats during development. Biochim. Biophys. Acta, 106: 540 (1965).

26. Zachman, R. D., Olson, E. B., Jr., Frantz, T. A., Bergseth, M. E., and Graver, S. N.: Respiratory distress syndrome with amniotic fluid lecithin/spingomyelin ratios greater than two (Abstract). Pediat. Res., 7: 167 (1973).

27. A hematocrit of greater than $0.05 \mathrm{cc} / 3 \mathrm{ml}$ is assumed to indicate excessive blood contamination as recommended by Gluck and Kulovich (13) in agreement with the later reports of Gibbons et al. (11) and Harding et al. (16) but in contrast to a report by Bryan (6).

28. Quick Scan, Helena Laboratories, Beaumont, Texas.

29. Physical evaluation of the infant was preferred (8); if this was not available, the obstetrician's estimation was used.

30. The acetone precipitation step recommended by Gluck et al. (12) was evaluated by determining the L/S ratios of $1402.0-\mathrm{ml}$ aliquots from the control pooled amniotic fluid. In 70 of these an acetone precipitation step was added to the described procedure. The average L/S ratios and their standard deviations were with the acetone precipitation step were 2.11 \pm 0.60 ; without the acetone precipitation step were $2.31 \pm 0.14$. The over fourfold increased variability introduced by the acetone precipitation step makes this procedure unacceptable.

31. The technical assistance of Margaret Bergseth and Thomas Frantz is gratefully acknowledged. In addition, we wish to thank Drs. John V. Hartline and Philip M. Farrell for their constructive help in preparing this manuscript.

32. This research was supported by National Institute of Health Grant 1 R01 HD05837-01A1 and the Wisconsin Perinatal Center, Southcentral Region, Madison, Wisc.

33. Requests for reprints should be addressed to: E. B. Olson, Jr., Ph.D., Hartford Neonatal Research Laboratories, St. Mary's Hospital Medical Center, 720 South Brooks St., Madison, Wisc. 53715 (USA).

34. Accepted for publication August 28, 1974. 